\title{
EXPLORING LITHIUM-ION INTENSITY AND DISTRIBUTION VIA A TIME-OF-FLIGHT SECONDARY ION MASS SPECTROSCOPY
}

\author{
Cheng-Kai ChiuHuang \\ Department of Mechanical and \\ Aerospace Engineering \\ North Carolina State University \\ Raleigh, North Carolina, USA \\ cchiuhu@ncsu.edu
}

\author{
Chuanzhen Zhou \\ Analytical Instrumentation Facility \\ College of Engineering, \\ North Carolina State University \\ Raleigh, North Carolina, USA \\ czhou@ncsu.edu
}

\author{
Hsiao-Ying Shadow Huang* \\ Department of Mechanical and \\ Aerospace Engineering \\ North Carolina State University \\ Raleigh, North Carolina, USA \\ hshuang@ncsu.edu
}

\begin{abstract}
For high rate-capability and low cost lithium-ion batteries, the prevention of capacity loss is one of major challenges facing by lithium-ion batteries today. During electrochemical processes, lithium ions diffuse from and insert into battery electrodes accompanied with the phase transformation, where ionic diffusivity and concentration are keys to the resultant battery capacity. In the current study, we first compare voltage vs. capacity curves at different C-rates $(1 \mathrm{C}, 2 \mathrm{C}, 6 \mathrm{C}, 10 \mathrm{C})$. Second, lithium-ion distributions and intensity are quantified via the Time-of-Flight Secondary Ion Mass Spectroscopy (ToFSIMS). The result shows that voltage vs. capacity relations are C-rate dependent and larger hystereses are observed in the higher C-rate samples. Detailed quantification of lithium-ion intensity for the 1C sample is conducted. It is observed that lithium-ions are distributed uniformly inside the electrode. Therefore, the current study provides a qualitative and quantitative data to better understand $\mathrm{C}$-rate dependent phenomenon of $\mathrm{LiFePO}_{4}$ battery cells.
\end{abstract}

\section{INTRODUCTION}

Lithium ion batteries play an important role in developing energy storage devices with high capacities. Among several developed cathode materials, the olivine-based lithium iron phosphate $\left(\mathrm{LiFePO}_{4}\right)$ with an orthorhombic crystal structure provide excellent characteristics for the application on EVs/PHEVs, such as good thermal stability, abundant iron ore resource, low raw material cost and high theoretical energy density $(170 \mathrm{mAh} / \mathrm{g})$ [1-3]. At room temperature, $\mathrm{LiFePO}_{4}$ works as a two-phase system during charging and discharging: a lithiated $\left(\mathrm{LiFePO}_{4}\right)$ and a delithiated $\left(\mathrm{FePO}_{4}\right)$ phases. The two-phase system of $\mathrm{LiFePO}_{4}$ could be explained by the Gibbs phase rule if we neglect the fluctuation in the millivolt scale: a single-phase region has the characteristic of varying potentials with different lithium concentrations, and a two-phase region would reveal a steady potential during charging/discharging. For $\mathrm{LiFePO}_{4}$, only the fluctuations in the scale of millivolt are observed at low C-rates [4]. However, this characteristic of the flat charging/discharging curve is C-rate dependent [5]. The absent of plateau zone in the charging/discharging curves under high C-rates indicates the change of lithium insertion/extraction mechanism accompanied with the phase transformation behavior. During the electrochemical process, lithium ions diffuse from and insert into battery electrodes. The investigation of lithium-ion intensity and distribution under different $\mathrm{C}$-rates could provide insight into how the lithium intercalation process changes within $\mathrm{LiFePO}_{4}$ particles.

To better detect lithium-ion intensity from surface to bulk for several electrode materials [7-13], Time-of-Flight Secondary Ion Mass Spectroscopy (ToF-SIMS) has been used, specifically to investigate the formation of Solid Electrolyte Interphase (SEI) layers. ToF-SIMS is a highly sensitive surface analytical technique that can be used to detect atoms and molecules even at low concentration down to ppm level [6]. The sample surface is bombard by a high energy $(\sim 25 \mathrm{keV})$ primary ion beams (e.g. $\left.\mathrm{Bi}^{+}\right)$. The emitted secondary ions from the sample will be then analyzed by calculating the flight time required to travel from the surface to the detector. A mass spectrum could be thus derived to compare the intensities of different atoms/molecules [6]. A depth profile showing the elemental intensity as a function of depth can be acquired using dual beam operation. While $\mathrm{Cs}^{+}$ion beam sputters the material to generate a crater, $\mathrm{Bi}^{+}$ion beam progressively analyzes the crater bottom. Therefore, in the current study, we utilize ToFSIMS techniques and extend its capability to investigate lithium-ion distribution and intensity profiles inside electrode

\footnotetext{
*Corresponding author
} 
materials. We first compare C-rate dependent voltage vs. capacity relations. Then relative mass/charge ratios of lithiumion are quantified via ToF-SIMS to investigate the lithium-ion intensity and distribution for a $1 \mathrm{C}$ fully discharged sample. The result of the experiment could be used as a baseline to compare with other lithium intensity at higher C-rates.

\section{METHOD}

\section{Charging/Discharging Experiment}

Commercial 26650 cylindrical $\mathrm{LiFePO}_{4}$ lithium ion batteries with a capacity of $2.5 \mathrm{Ah}$ are used in the current study. The charging/discharging experiments are conducted via an Arbin BT2000 cycler (Arbin Instrument, College Station, TX) which is located at North Carolina State University. The C-rates of $1 \mathrm{C}, 2 \mathrm{C}, 6 \mathrm{C}, 10 \mathrm{C}(1 \mathrm{C}=2.5 \mathrm{~A})$ are chosen and compared in this study. By referring to the experimental protocol of the automotive application batteries [14], the following steps are used to conduct the charging/discharging experiment: (1) Galvanostatically charge to $3.6 \mathrm{~V}$ at constant current $\mathrm{I}=2.5 \mathrm{~A},(2)$ convert to potentiostatic charging at constant voltage $\mathrm{V}=3.6 \mathrm{~V}$ until the current drops to $\mathrm{C} / 20(0.125 \mathrm{~A})$. The purpose of this step is to ensure the battery is fully charged. (3) Discharge the batteries at different C-rates until the voltage drops to the recommended lower bound of the working voltage $(2 \mathrm{~V})$, and (4) galvanostatically charge the battery at different C-rates to $3.6 \mathrm{~V}$. The steps (1) to (4) are repeated three times in order to ensure that a repeatable voltage-time curve is obtained.

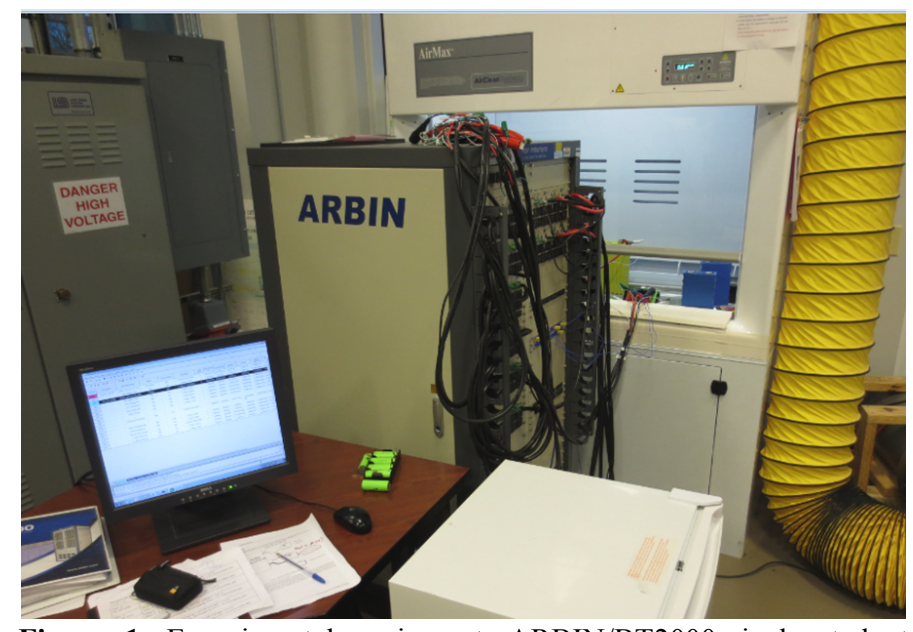

Figure 1. Experimental equipment, ARBIN/BT2000, is located at NCSU for the battery charging/discharging test with the maximum current capacity of \pm 50 A.

\section{ToF-SIMS}

(1) Sample preparation

In general, ToF-SIMS is not a quantitative analytical method because many factors such as the ionization probability, and matrix effect are varied for different species and samples. Thus, we first select a $1 \mathrm{C}$ fully discharged sample as our baseline data for the comparisons. After being discharged to $0 \mathrm{~V}$ with $1 \mathrm{C}$, the battery is disassembled in the ambient air and the $\mathrm{LiFePO}_{4}$ cathode material is unrolled, as shown in Figure 2. To evaluate the effects of air on $\mathrm{LiFePO}_{4}$ sample, repeated studies would be conducted in the glove box within a nitrogen environment (less than $0.1 \mathrm{ppm}$ of both oxygen and water.) The said glove box is located in the Nanofabrication Facility at NCSU. $\mathrm{LiFePO}_{4}$ is deposited on the aluminum foil and the carbon anode material is deposited on the copper foil. The sample size of approximately $1 \mathrm{~mm} \times 1 \mathrm{~mm}$ is removed from the unrolled strip for ToF-SIMS analyses.

\section{(2) TOF-SIMS analysis}

ToF-SIMS analyses in this study are conducted by using an ION TOF ToF-SIMS V (ION TOF, Inc. Chestnut Ridge, NY) instrument equipped with a $\mathrm{Bi}_{n}{ }^{\mathrm{m}+}(\mathrm{n}=1-5, \mathrm{~m}=1,2)$ liquid metal ion gun and a $\mathrm{Cs}^{+}$sputtering ion gun. Both the $\mathrm{Bi}$ and $\mathrm{Cs}$ ion columns are oriented at $45^{\circ}$ with respect to the sample surface normal. The instrument vacuum system consists of a load lock for rapid sample loading connected by a gate valve to the analysis chamber. The analysis chamber pressure is maintained at or below $5.0 \times 10^{-9}$ mbar to avoid contamination of the surfaces to be analyzed. For the ToF-SIMS depth profile acquisition, $\mathrm{Cs}^{+}$sputtering ion beam is rastered over a $80 \times 80$ $\mu \mathrm{m}$ area to generate a crater, and a $25 \mathrm{keV} \mathrm{Bi}_{3}{ }^{+}$analysis beam is used to analyze $10 \mu \mathrm{m} \times 10 \mu \mathrm{m}$ area on the crater bottom. The two ion beams operate alternatively to reveal elemental intensity as a function of depth.

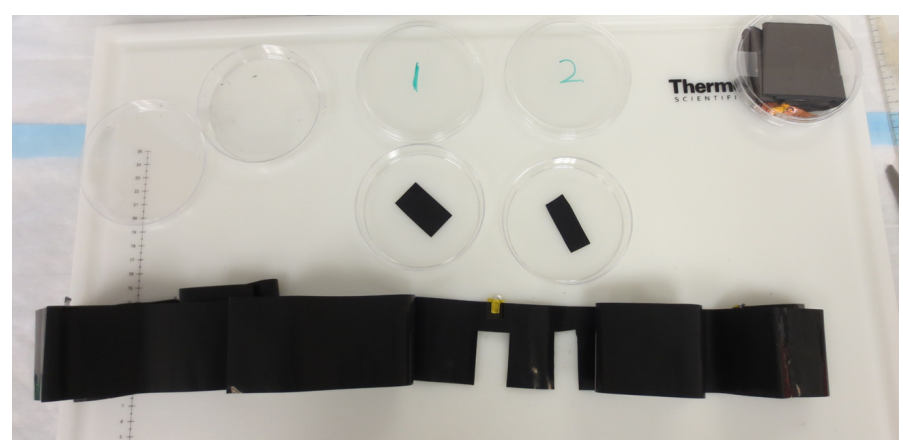

Figure 2. The unrolled $\mathrm{LiFePO}_{4}$ stripe taken from a $1 \mathrm{C}$ fully discharged cylindrical battery. $\mathrm{LiFePO}_{4}$ cathode material is deposited on the aluminum foil.

\section{RESULTS AND DISCUSSION}

\section{Charging/Discharging Curves}

The voltage vs. capacity curves for different C-rates (1C, 2C, 6C and 10C) are shown in Figure 3. The discharging curves are represented in solid lines while the charging curves are shown in dashed lines. In Figure 3, the hysteresis between charging and discharging curves for all $\mathrm{C}$-rates are observed. The voltage gap (between charging and discharging) increases with an increasing C-rate. For example, considering the 
location of the capacity $=0.015(\mathrm{Ah} / \mathrm{g})$, the voltage gaps are approximately $0.18(\mathrm{~V}), 0.25(\mathrm{~V}), 0.45(\mathrm{~V})$, and $0.68(\mathrm{~V})$ for $1 \mathrm{C}, 2 \mathrm{C}, 6 \mathrm{C}$ and $10 \mathrm{C}$, respectively (Figure 3). The trend of these quantitative data is consistent with the observation by Dryer et al. [15]. Besides, due to the characteristic of the twophase system for $\mathrm{LiFePO}_{4}$ material, flat voltage curves are noticed between the capacity of 0.005 and $0.025(\mathrm{Ah} / \mathrm{g})$ for all $\mathrm{C}$-rates. However, by comparing the voltages of discharging curves in the millivolt scale, the voltage curves in this section are not perfectly horizontal, as shown in the inset of Figure 3. An increasing dropping-slope of the curves is observed with higher C-rate, and a stronger voltage fluctuation for the $10 \mathrm{C}$ sample is noticed, suggesting that an extra over-potential is required to overcome the energy barrier during the phase transformation of $\mathrm{LiFePO}_{4}$ [15-17]. That is, the voltage fluctuation indicates a slightly different lithium insertion mechanism, e.g. rather than a well-known first-order phase transformation or a layer-by layer phase transformation. Due to a higher driving force for the $10 \mathrm{C}$ sample, lithium ions could possibly insert into multiple particles at the same time [15]. This might cause a different lithium concentration profile along the sample thickness or a different lithium-ion distribution on the plane normal to the direction of the sample thickness.

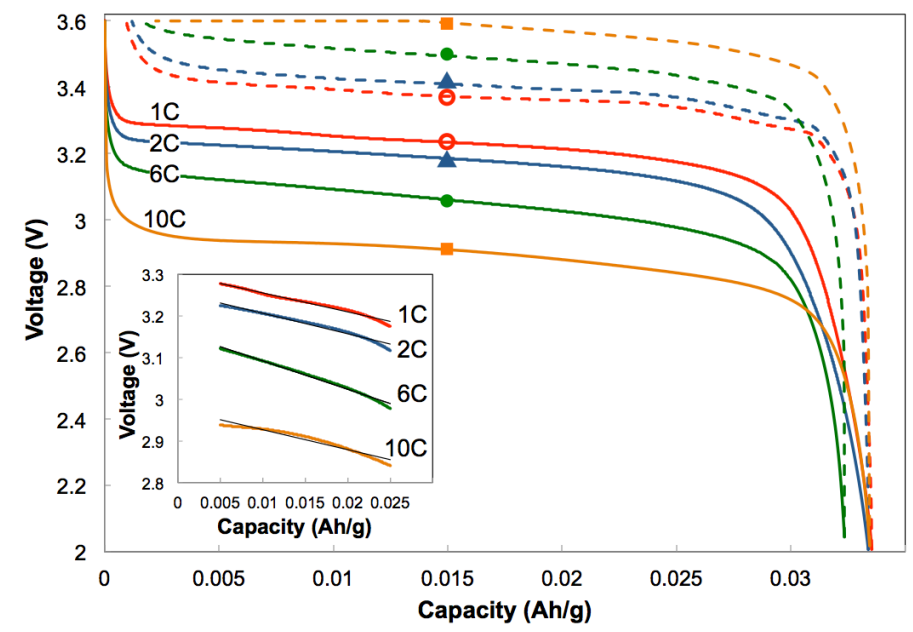

Figure 3. Voltage vs. capacity curves at different C-rates (1C, 2C, 6C and $10 \mathrm{C}$ ). The voltage gap increases with an increasing C-rate. Inset: linear trend lines between the capacities of 0.005 and 0.025 . The dropping-slope increase from $1 \mathrm{C}$ to $6 \mathrm{C}$. A more noticeable voltage fluctuation is observed in the $10 \mathrm{C}$ sample.

\section{ToF-SIMS}

Depth profile acquisition using ToF-SIMS is destructive due to the removal of sample material during the analysis. Figure 4 shows the sample surface before and after sputtering. The crater size generated by $\mathrm{Cs}^{+}$ion beam is $80 \mu \mathrm{m} \times 80 \mu \mathrm{m}$ and to avoid crater effect, a representative sample with a $10 \mu \mathrm{m}$ $\mathrm{x} 10 \mu \mathrm{m}$ area is analyzed by $\mathrm{Bi}_{3}{ }^{+}$ion beam. The result of ToFSIMS positive ion mass spectrum integrated from the surface to crater bottom is shown in Figure 5, where two peaks of isotopes of lithium $\left({ }^{6} \mathrm{Li}:{ }^{7} \mathrm{Li}=7.59 \%: 92.41 \%\right.$ in nature $)$ are identified. Based on the intensity, ${ }^{6} \mathrm{Li}$ is used in the current study because the intensity of ${ }^{7} \mathrm{Li}$ exceeds the limit of the detector. $\mathrm{Fe}^{+}$or $\mathrm{P}^{+}$are also observed in the positive ion mass spectrum (not shown). The intensity of lithium-ion is very high compared to other atoms such as $\mathrm{Fe}^{+}$or $\mathrm{P}^{+}$in the $\mathrm{LiFePO}_{4}$ material sample due to much higher secondary ion yield of $\mathrm{Li}$.
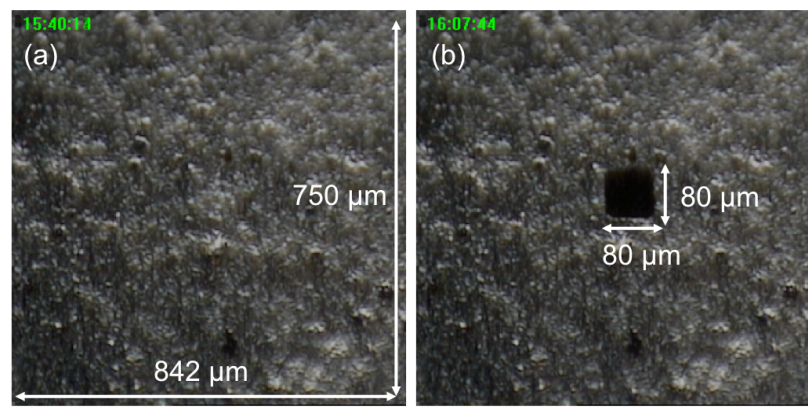

Figure 4. Images of the sample surface (a) before and (b) after the sputtering process. Window view size is $842 \times 750 \mu \mathrm{m}$. The crater size is $80 \mu \mathrm{m} \times 80 \mu \mathrm{m}$, and the analysis area is $10 \mu \mathrm{m} \times 10 \mu \mathrm{m}$ in the middle to avoid the crater effects.

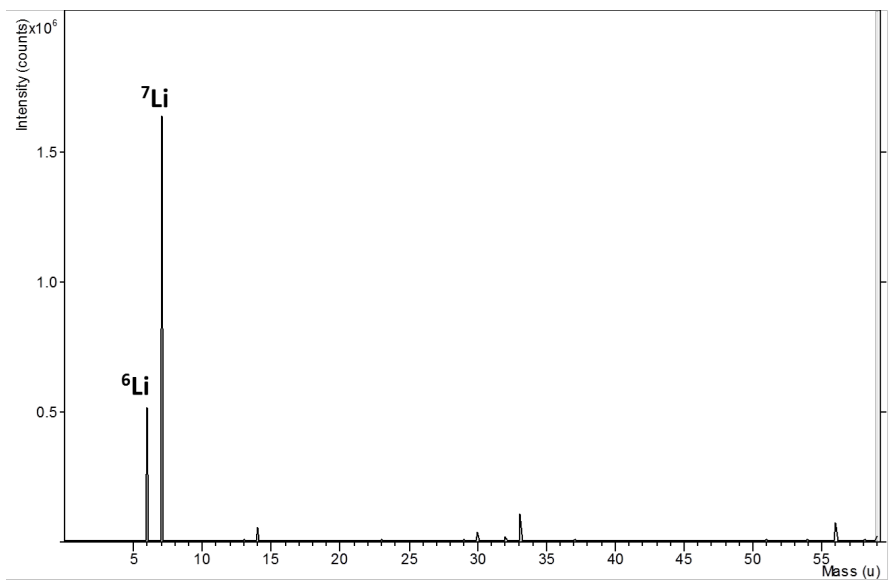

Figure 5. ToF-SIMS positive ion mass spectrum integrated from the sample surface to the crater bottom. The first two peaks represent ${ }^{6} \mathrm{Li}^{+}$ and ${ }^{7} \mathrm{Li}^{+}$. The intensity of ${ }^{6} \mathrm{Li}^{+}$is chosen in this study due to the saturation of ${ }^{7} \mathrm{Li}^{+}$.

\section{Depth profile analysis}

Figure 6 shows the positive ion depth profiles of ${ }^{6} \mathrm{Li}^{+}, \mathrm{Fe}^{+}$ and $\mathrm{P}^{+}$for a fully discharged $1 \mathrm{C} \mathrm{LiFePO}_{4}$ sample. As the sputtering time progresses, the intensity of elements is recorded along the thickness of the sample. After sputtering reaches the equilibrium at about 200 s, the lithium-ion intensity becomes very stable. Because the intensity of detected secondary ions is affected by their ionization probability as shown here, the intensities do not represent their concentration. To establish a relationship between the intensity and the concentration of each element, an appropriate sample to define the baseline is 
required. For example, here we present a lithium-ion intensity profile for the $1 \mathrm{C} \mathrm{LiFePO}_{4}$ sample, a qualitative and a semiquantitative value for samples with higher $\mathrm{C}$-rates could be derived. An ongoing project is currently carried on to quantify and qualify such phenomenon, and it is not presented in the current study. Furthermore, during charging/discharging processes, lithium ions are inserted and extracted from cathode materials while iron atoms remain inside the crystal. Thus, we could also use the intensity of $\mathrm{Fe}^{+}$to compare with the intensity of lithium. Two intensities of $\mathrm{Fe}^{+}$are presented in Figure 6: one is with its original detected intensity along the thickness of the sample, and another intensity is factored by four to provide a better comparison. As it can be seen, the intensity variation of $\mathrm{Fe}^{+}$is similar and correlated to that of ${ }^{6} \mathrm{Li}^{+}$. Thus, we could possibly assume that the particles are almost fully filled with lithium and become $\mathrm{LiFePO}_{4}$ phase in this fully discharged sample.

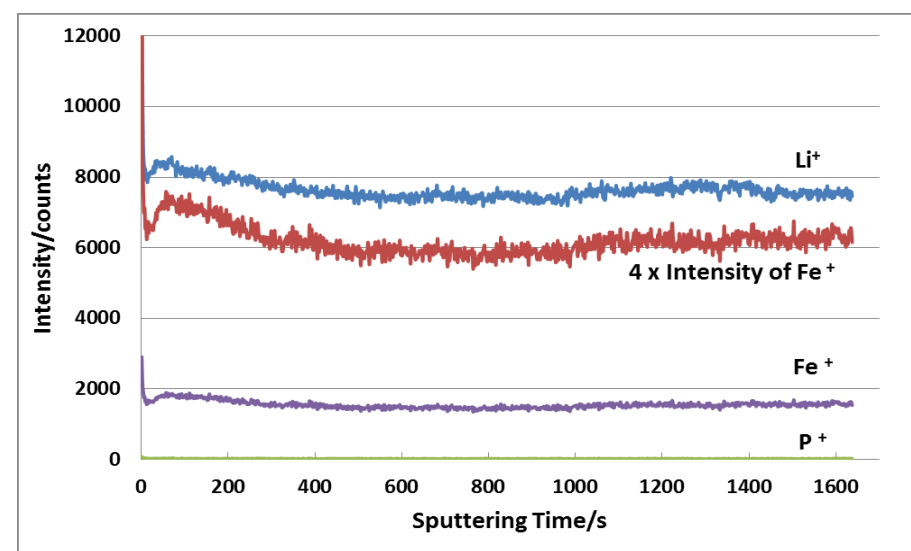

Figure 6. ToF-SIMS positive ion depth profiles of a fully discharged $1 \mathrm{C} \mathrm{LiFePO}_{4}$ cathode material sample.

\section{D intensity distribution}

Figure7 shows the lithium intensity and the distribution on the plane normal to the direction of the thickness. The field of the view is $10 \mu \mathrm{m} \times 10 \mu \mathrm{m}$ divided into $128 \times 128$ pixels. Assuming the average spherical particle size of $100 \mathrm{~nm}$, the area contains approximately $10,000 \quad \mathrm{LiFePO}_{4}$ particles. Therefore, each pixel represents about 0.6 particles: $10,000 /(128 \times 128)$. Note that lithium $\left({ }^{6} \mathrm{Li}^{+}\right)$and iron $\left(\mathrm{Fe}^{+}\right)$ intensities are distributed very uniformly in this rastered area (Figure 7a-b). We could compare the intensity variation along the $\mathrm{x}$-axis at different locations along the $\mathrm{y}$-axis. The position of each pixel is defined by a coordinate system $(\mathrm{X}, \mathrm{Y})$ ranging from $(0,0)$ to $(127,127)$. Figure 7 (c) and (d) show Li-ion intensity along $\mathrm{x}$-axis when $\mathrm{Y}=0$ and $\mathrm{Y}=63$. It can be seen that in both cases the intensities mainly fluctuate between 320 and 400 counts. No obvious increasing or decreasing trend line is observed. This fluctuation is possibly caused by the nonuniform particle size distribution in the sample. Sample with a more uniform particle size distribution should show less fluctuation in ion intensities. Further SEM or TEM experiments need to be performed to illustrate actual particle size in the sample. The ratio of $\mathrm{Li}$ to $\mathrm{Fe}$ of $1 \mathrm{C}$ fully discharging sample could be used as the baseline to compare lithium intensities at different C-rates or at different State of Discharge (SOD) to investigate the $\mathrm{C}$-rate dependent lithium insertion/extraction mechanism.
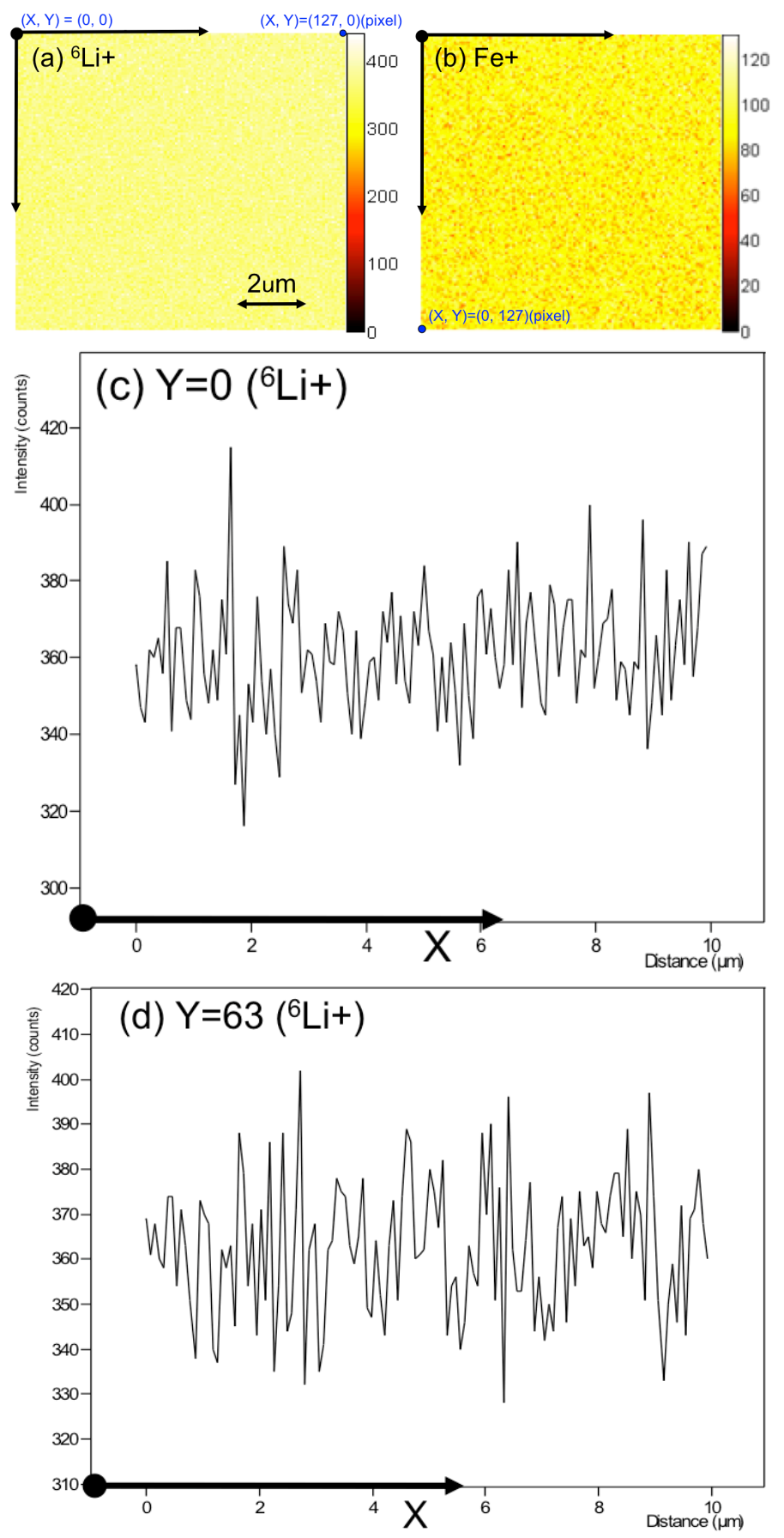

Figure 7. ToF-SIMS images of (a) ${ }^{6} \mathrm{Li}+$ and (b) $\mathrm{Fe}+$. (c) ${ }^{6} \mathrm{Li}+$ intensity profile along the $\mathrm{X}$ direction at $\mathrm{Y}=0$ (d) ${ }^{6} \mathrm{Li}+$ intensity profile along the $\mathrm{X}$ direction at $\mathrm{Y}=63$. 


\section{CONCLUSION}

In the present study, we compare voltage vs. capacity curves at different $\mathrm{C}$-rates $(1 \mathrm{C}, 2 \mathrm{C}, 6 \mathrm{C}, 10 \mathrm{C})$ for $\mathrm{LiFePO}_{4}$ materials. The voltage gap between charging and discharging curves increases with the increasing of $\mathrm{C}$-rate. The voltage fluctuation for $10 \mathrm{C}$ is more noticeable comparing to $1 \mathrm{C}, 2 \mathrm{C}$ and $6 \mathrm{C}$ samples. It is suggested that for a multi-particle system, lithium ions insert into many particles simultaneously, rather than a particle-by-particle lithiation process observed at lower C-rates. Studies by ToF-SIMS techniques show that the lithium-ion distribution within a 1C fully discharged sample is very uniform. Similar trend of the intensity variation of the depth profiles for ${ }^{6} \mathrm{Li}^{+}$and $\mathrm{Fe}^{+}$are observed, and it indicates that the lithium-ions are almost fully inserted inside the materials. The results from the current study could be used as the baseline to compare with other samples prepared under different C-rates. Our future work will include ToF-SIMS analysis for high $\mathrm{C}$-rate samples (6C and 10C).

\section{ACKNOWLEDGEMENT}

We are grateful to the Advanced Transportation Energy Center, a part of the NSF funded Future Renewable Electric Energy Delivery and Management (FREEDM) systems center at NCSU for the use of advanced Arbin BT2000 cycler. This work is partially supported by the NC Space Grant and Sigma Xi Grants-in-Aid of Research Program. C.-K. C.H. acknowledges support by the Taiwan Study Abroad Scholarship.

\section{REFERENCE}

[1] Yuan, L., Wang, Z., Zhang, W., 2011, "Development and Challenges of $\mathrm{LiFePO} 4$ Cathode Material for Lithium-Ion Batteries," Energy \& Environmental Science, 4(2) pp. 269-284.

[2] Park, M., Zhang, X., Chung, M., 2010, "A Review of Conduction Phenomena in Li-Ion Batteries," Journal of Power Sources, 195(24) pp. 7904-7929.

[3] Zhang, W., 2011, "Structure and Performance of LiFePO(4) Cathode Materials: A Review," Journal of Power Sources, 196(6) pp. 2962-2970.

[4] Meethong, N., Kao, Y., Tang, M., 2008, "Electrochemically Induced Phase Transformation in Nanoscale Olivines Li1xMPO4 (M = Fe, Mn)," Chemistry of Materials, 20(19) pp. 6189-6198.

[5] Kang, B., and Ceder, G., 2009, "Battery Materials for Ultrafast Charging and Discharging," Nature, 458(7235) pp. 190-193.

[6] Belu, A., Graham, D., and Castner, D., 2003, "Time-ofFlight Secondary Ion Mass Spectrometry: Techniques and Applications for the Characterization of Biomaterial Surfaces," Biomaterials, 24(21) pp. 3635-3653.

[7] Castle, J. E., Decker, F., Salvi, A. M., 2008, "XPS and TOF-SIMS Study of the Distribution of Li Ions in Thin Films of Vanadium Pentoxide After Electrochemical Intercalation," Surface and Interface Analysis, 40(3-4) pp. 746-750.

[8] Fedorkova, A., Orinakova, R., Orinak, A., 2011, "Electrochemical and TOF-SIMS Investigations of PPy/PEGModified LiFePO4 Composite Electrodes for Li-Ion Batteries," Solid State Sciences, 13(5) pp. 824-830.

[9] Hong, T. E., Jeong, E. D., Baek, S. R., 2012, "Nano SIMS Characterization of Boron- and Aluminum-Coated LiNi1/3Co1/3Mn1/3O2 Cathode Materials for Lithium Secondary Ion Batteries," Journal of Applied Electrochemistry, 42(1) pp. 41-46.

[10] Li, J., Maurice, V., Swiatowska-Mrowiecka, J., 2009, "XPS, Time-of-Flight-SIMS and Polarization Modulation IRRAS Study of $\mathrm{Cr} 2 \mathrm{O} 3$ Thin Film Materials as Anode for Lithium Ion Battery," Electrochimica Acta, 54(14) pp. 37003707.

[11] Li, J., Swiatowska, J., Maurice, V., 2011, "XPS and ToFSIMS Study of Electrode Processes on Sn-Ni Alloy Anodes for Li-Ion Batteries," Journal of Physical Chemistry C, 115(14) pp. 7012-7018.

[12] Li, J., Swiatowska, J., Seyeux, A., 2010, "XPS and ToFSIMS Study of Sn-Co Alloy Thin Films as Anode for Lithium Ion Battery," Journal of Power Sources, 195(24) pp. 8251-8257. [13] Swiatowska-Mrowiecka, J., Martin, F., Maurice, V., 2008, "The Distribution of Lithium Intercalated in V2O5 Thin Films Studied by XPS and ToF-SIMS," Electrochimica Acta, 53(12) pp. 4257-4266.

[14] Zhang, Y., Wang, C., and Tang, X., 2011, "Cycling Degradation of an Automotive $\operatorname{LiFePO}(4)$ Lithium-Ion Battery," Journal of Power Sources, 196(3) pp. 1513-1520.

[15] Dreyer, W., Jamnik, J., Guhlke, C., 2010, "The Thermodynamic Origin of Hysteresis in Insertion Batteries," Nature Materials, 9(5) pp. 448-453.

[16] Van der Ven, A., Garikipati, K., Kim, S., 2009, "The Role of Coherency Strains on Phase Stability in $\operatorname{Li}(x) \mathrm{FePO}(4)$ : Needle Crystallites Minimize Coherency Strain and Overpotential," Journal of the Electrochemical Society, 156(11) pp. A949-A957.

[17] Malik, R., Zhou, F., and Ceder, G., 2011, "Kinetics of Non-Equilibrium Lithium Incorporation in LiFePO(4)," Nature Materials, 10(8) pp. 587-590. 